\title{
A 2D Finite Element Study on the Flow Pattern and Temperature Distribution for an Isothermal Spherical Furnace with the Aperture
}

\author{
Sudhakar Matle, Subbiah Sundar \\ Mathematics, IIT Madras, Chennai, India \\ Email: iitsudha@gmail.com, slnt@iitm.ac.in
}

Received October 11, 2012; revised November 12, 2012; accepted November 21, 2012

\begin{abstract}
Calibration of radiation thermometers is one of the important research activities in the field of metrology. Many researchers in recent times have conducted numerical simulations on the calibration furnace to understand and overcome the experiment limitations. This paper presents a 2D numerical free convective study on the calibration furnace with the aperture using finite element method. The focused issues here are: aspect ratio effect on the flow pattern and temperature fields, heat transfer mechanism in the aperture zone as well as in hump regime. It is concluded that flow and temperature fields follow the same behavior in the hump regime as well as in the aperture zone. Also, it concluded that penetrative convection is more dominant for the enclosure of high aspect ratio.
\end{abstract}

Keywords: Calibration Furnace; Thermal Penetration; Rayleigh Number; Aspect Ratio

\section{Introduction}

Temperature measurement of the very hot objects by means of contact thermocouple is not always handy. For this purpose, radiation thermometer is used to measure temperature in terms of radiation emitted by the hot object. Radiation thermometer is a temperature measurement instrument and measures temperature of the very hot object from a distance. For accuracy of the instrument, it must be calibrated. Calibration is an act of adjusting instrument by comparison against any standard surface. The Saturn (a hollow spherical cavity) is one of such standards used in radiation thermometer calibration [1]. For details on calibration furnace and the finite element modeling to study experiment limitations, one can refer authors' previous work [2].

Many research efforts have been done finite element modeling $[3,4]$ of calibration furnaces, industrial furnaces, heated spheres $[5,6]$ and solar cavity receivers for quality equipment. Recently, Oluwole et al. [7] studied the flow patterns in two salt bath furnaces using finite element analysis. The implications of the heat flows on long term stability of furnace performance were evaluated. Khoei et al. [8] developed a finite element model that is employed to simulate the furnace rotation and analyze energy flows inside the furnace. Also, finite element, stream function vorticity solutions for steady state incompressible Navier Stokes equations are derived in papers $[9,10]$.

Natural convection in fluid filled enclosures [11,12] with heating bottom and sides [13] have been studied extensively. Sarris et al. [14] studied numerically natural convection in a rectangular enclosure with heating from the top wall with all others insulated by varying Rayleigh number from $10^{2}$ to $10^{8}$ and the aspect ratio from 0.5 to 2 . Recently, Hartlep et al. [15] performed simulations of Rayleigh-Benaurd convection in a large box of aspect ratio 10 over a range of Rayleigh and Prandtl numbers provide important insight into the choice of the aspect ratio. In another study, Lee et al. [16] stressed the need for very large aspect ratio domains by studying the natural convection in a horizontal fluid layer with a periodic array of internal square cylinders.

Finite element mesh is a part of numerical simulation study. Sensitivity analysis for parameter dependent optimization problems is an active area of research in the context of solution of partial differential equations. Becker and Vexler $[17,18]$ investigated sensitivity of the mesh based on relative condition numbers which describe the influence of small changes in measurements on the value of interest functional.

This paper addresses a 2D numerical free convective study on the calibration furnace with the aperture. In the next section, the $2 \mathrm{D}$ problem, governing equations and the corresponding boundary conditions are systematically discussed. In Section 3, mathematical formulation of 2D 
problem and then numerical simulation schematically presented. Section 4 presents precise results on flow and temperature fields near the proximity of the furnace surface and in the aperture plane.

\section{Statement of the Problem}

The problem is addressed in the context of temperature distribution and 2D flow pattern for the furnace in three various types of enclosures, but, the method, ideas and results can be compared to enclosure of any dimension and $3 \mathrm{D}$ model. The qualitative behavior of flow and heat transfer is the same for a general 2D model and 3D model due to bi-axial symmetry of the sphere. For this purpose, a furnace slice, obtained by cutting along the equatorial line, kept in an enclosure of two dimensions is considered as a computational domain for the present study. The coordinates chosen for the enclosure computational domain of aspect ratio 1 are $\mathrm{E}(0,0), \mathrm{F}(0.3,0)$, $\mathrm{G}(0.3,0.3)$ and $\mathrm{H}(0,0.3)$. The furnace dimensions are taken from the experiment and it is shown in Figure 1. The enclosure is filled with air to keep maintain experiment conditions.

\section{Mathematical Model}

The computational domain $(\Omega)$ is assumed to be union of the two sub domains $\Omega_{1}$ (gas) and $\Omega_{2}$ (solid). The schematic sketch of the computational domain is shown in Figure 1. Concentric sub domains are established with settings from the outermost to innermost are: stainless steel $(44.5 \mathrm{~W} / \mathrm{mK})$, ceramic $\mathrm{SiO}_{2}(1.4$ $\mathrm{W} / \mathrm{mK})$, concrete $(1.8 \mathrm{~W} / \mathrm{mK})$, copper $(400 \mathrm{~W} / \mathrm{mK})$ and ceramic $\left(\mathrm{SiO}_{2}\right)$. The value in the parentheses represents thermal conductivity of the corresponding material. The innermost sub domain is filled with air at Boussinesq. $20^{\circ} \mathrm{C}$.

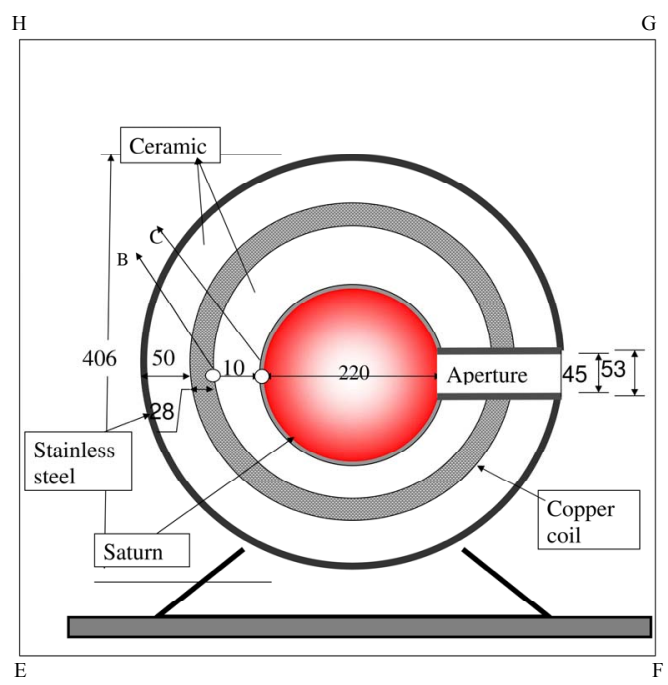

Figure 1. Schematic sketch of the computational domain.
The flow is incompressible air and two dimensional, there is no viscous dissipation, gravity acts in vertical direction, air properties are constant and density variations are neglected except the buoyancy term. Continuity and momentum equations are defined in the air domain $\Omega_{1}$ as follows:

$$
\begin{aligned}
& \frac{\partial u}{\partial x}+\frac{\partial v}{\partial y}=0 \\
& u \frac{\partial u}{\partial x}+v \frac{\partial u}{\partial y}=-\frac{\partial p}{\partial x}+v \Delta u \\
& u \frac{\partial v}{\partial x}+v \frac{\partial v}{\partial y}=-\frac{\partial p}{\partial y}+v \Delta v+g \beta\left(T-T_{a m b}\right)
\end{aligned}
$$

The heat energy equation is defined in the entire computational domain $(\Omega)$ to study the heat transport equation is as follows.

$$
\begin{aligned}
& u \frac{\partial T}{\partial x}+v \frac{\partial T}{\partial y}=\alpha \Delta T \text { in } \Omega_{1} \\
& \nabla^{2} T+\frac{Q}{k}=0 \text { in } \Omega_{2}
\end{aligned}
$$

where $Q=0$ everywhere except at the heat source region.

\section{Mathematical Formulation}

The steady state incompressible Navier-Stokes equations in velcoty-vector potential formulation are presented as

$$
\begin{aligned}
& (u \cdot \nabla) \omega=v \Delta \omega+g \beta\left(T-T_{a m b}\right) \\
& \Delta \psi=\omega
\end{aligned}
$$

with no slip boundary conditions

$$
\psi=0, \frac{\partial \psi}{\partial n}=0
$$

while the velocity is given by

$$
u=\nabla^{\perp} \psi, \nabla^{\perp}=(\partial y,-\partial x)
$$

The stream function, and hence the velocity, is then calculated from $\Delta \psi=\omega$.

Weak form of Equations (3)-(5) is as follows: find $\psi \in H_{0}{ }^{1}\left(\Omega_{1}\right)$ and $\omega \in H^{1}\left(\Omega_{1}\right)$ such that

$$
\begin{aligned}
& \langle\nabla \phi, w \bar{u}\rangle=v\langle\nabla \phi, \nabla \omega\rangle+g \beta\left\langle\phi, T-T_{\text {amb }}\right\rangle \forall \phi \in H_{0}^{1} \\
& \langle\nabla \phi, \nabla \psi\rangle=-\langle\phi, \omega\rangle \forall \phi \in H^{1}
\end{aligned}
$$

where $\langle\cdot\rangle$, denotes the standard inner product on $\Omega_{1}$ and $\|\cdot\|$ for $L_{2}$ norm. Let $X_{h}^{2}$ be the standard continuous finite element space with the 2nd degree polynomial on each element of a triangulation. Let $X_{0, h}^{2}$ be the subspace of $X_{h}^{2}$ with zero boundary values. For the Lagrangian finite element space,

$\operatorname{dim}\left(X_{0, h}^{2}\right)=N_{i}$ and $\operatorname{dim}\left(X_{h}^{2}\right)=N_{i}+N_{b}$ where in $N_{i}$ 
the number of interior nodes and $N_{b}$ is number of boundary nodes. Then the finite element approximation is as follows: find $\omega_{h} \in X_{h}^{2}$ and $\psi_{h} \in X_{0, h}^{2}$ such that

$$
\begin{aligned}
& \left\langle\nabla \phi_{h}, \omega_{h} \bar{u}_{h}\right\rangle=v\left\langle\nabla \phi_{h}, \nabla \omega_{h}\right\rangle \quad \forall \phi_{h} \in X_{0, h}^{2} \\
& \left\langle\nabla \phi_{h}, \nabla \psi_{h}\right\rangle=-\left\langle\phi_{h}, \omega_{h}\right\rangle, \quad \forall \phi_{h} \in X_{h}^{2}
\end{aligned}
$$

The velocity $\bar{u}_{h}$ is obtained from the stream function through

$$
\bar{u}_{h}=\nabla^{\perp} \psi_{h}
$$

Clearly the velocity $\bar{u}_{h}$ satisfies the divergence free condition everywhere and the normal velocity $\bar{u}_{h} \cdot \bar{n}$ is continuous across element boundary. The absolute value of the extreme stream function is evaluated at the stagnant fluid, i.e.

$$
\left|\psi_{\text {ext }}\right|=\left.\omega\right|_{u=0, v=0} \times \text { element area }
$$

The heat transfer equation defined on $\Omega_{1}$ as follows: find $T_{h} \in Y_{h}^{2}$ such that

$$
\left\langle\bar{u}_{h} T_{h}, \nabla \bar{\tau}_{h}\right\rangle=\alpha\left\langle\nabla \bar{\tau}_{h}, \nabla T_{h}\right\rangle \forall \bar{\tau}_{h} \in Y_{h}^{2}
$$

where $Y_{h}^{2}$ is a finite element space of second degree polynomial defined on $\Omega_{1}$.

On $\Omega_{2}$, heat transfer equation is defined as follows: find $T_{h} \in Y_{h}^{2}$ such that

$$
\left\langle T_{h}, \nabla \bar{\tau}_{h}\right\rangle=\left\langle Q / k, \nabla \bar{\tau}_{h}\right\rangle \forall \bar{\tau}_{h} \in Y_{h}^{2}
$$

A quadratic triangular finite element mesh of maximum element size 0.02 is chosen for the current numerical study. Maximum element size is defined as the ratio of maximum edge length to the unit length of the element.

Sensitivity of the mesh is examined for two different meshes composed of 9989 nodes and 10,667 nodes based on relative condition numbers. From Table 1, measurements at $k_{m}$ (the point on the cavity surface exactly opposite to the aperture) have more influenced than $k_{b}$ (point at the cavity bottom) and $k_{t}$ (point at the cavity top). It is reasonable to say that at $k_{m}$, the solution gradient is very high due to curvature. Therefore, the mesh is refined locally towards direction normal to $k_{m}$.

\section{Results and Discussion}

As the heat passes through ceramic, there is a rapid decrease in temperature but not zero and it is shown in

Table 1. Relative condition numbers for point measurements on locally refined meshes.

\begin{tabular}{cccc}
\hline $\mathrm{N}$ & $k_{b}$ & $k_{m}$ & $k_{t}$ \\
\hline 9989 & 0.000134 & 0.000133 & 0.00012 \\
10,667 & 0.000146 & 0.000114 & 0.000114 \\
\hline
\end{tabular}

Figure 2. In order to better understand heat transfer phenomena in an enclosure, the profile near the proximity of the surface top is studied. The profile is sinusoidal in the interval range $0.3<x<0.5$ indicates the possibility of occurrence of Rayleigh-Benaurd convection in the region above surface top.

Three enclosures of aspect ratio 0.5 (narrow), 1 and 1.5 (wide) are chosen for current numerical investigation. Also, it is important to notify the assumption that the dimensionless temperature $\theta$ is 1 at the heating coil $\mathrm{B}$ $(0.26,0.4)$ only when cavity attains the experiment temperature $T_{s}=409.8^{\circ} \mathrm{C}$ at $\mathrm{C}(0.29,0.4)$. No radiation effects are considered in the current study. Typical flow and temperature fields, mechanism of heat transfer and thermal penetration in the aperture region are thoroughly discussed by varying Rayleigh numbers from $10^{4}$ to the high Rayleigh number achieved.

\subsection{Flow and Temperature Fields}

For the aspect ratio 1, flow pattern is investigated based on vorticity values in a stagnant fluid from Rayleigh number $10^{4}$ to the high Rayleigh number achieved.

At the Rayleigh number $10^{4}$, the flow is not rotational at the region where the boundary layer entrains into the main stream. Therefore, stream function satisfies Laplace equation. Hence the strength of circulation at the low Rayleigh number is $0.000064(x=0.4, y=0.604)$. At the Rayleigh number $10^{5}$, strength of the circulation is $0.00068(x=0.485, y=0.585)$. At this stage, convective flow cell is formed rotating in an anti-clock wise direction. At the high Rayleigh number $8.2 \times 10^{5}$, strength of the circulation is $0.00188(x=0.43, y=0.601)$ and therefore both clockwise and anti-clockwise flow pattern predicted with high velocity. Thus, as expected circulation strength increase with the Rayleigh number and the coordinate shifts from close to the hot cell towards inside, (in an anti-clockwise sense) i.e. the flow penetrates into the enclosure more and more with an increasing Rayleigh number.

In the aperture plane, strength of circulation is $0.0000145(x=0.51, y=0.4)$ at the Rayleigh number $10^{4}$.

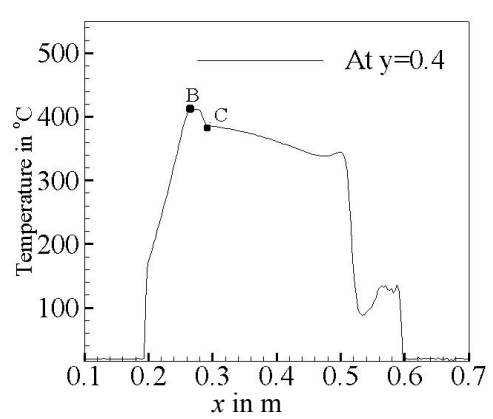

Figure 2. Temperature profile at the vertical midpoint along horizontal layer of cross section. 
Therefore, only weak cells are predicted in between $x=$ 0.51 and $x=0.6$. As the Rayleigh number increases to $10^{5}$, the corresponding circulation strength is $0.00252(x=$ $0.51, y=0.4)$. Thus, circulation strength increases with the Rayleigh number and the cold fluid penetrates into the aperture more and more with an increasing Rayleigh number. Also, there is one more point $(x=0.58, y=0.4)$ at which fluid is stagnant. The corresponding circulation strengths are $0.01\left(\mathrm{Ra}=10^{4}\right), 0.064\left(\mathrm{Ra}=10^{5}\right)$ and 0.42 $\left(\mathrm{Ra}=8.2 \times 10^{5}\right)$. It indicates there is a strong convective cell around that point.

For the aspect ratio 0.5 , circulation strengths near the hump regime are $0.00282(0.435,0.6), 0.0069(0.49$, $0.582)$ and $0.05775(0.4,0.603)$ for Rayleigh numbers $10^{4}, 10^{5}$ and $4.9 \times 10^{5}$ respectively. At the Rayleigh number $10^{4}$, penetration of the flow is not vertical and hence the low flow velocity is predicted in the regime. As the Rayleigh number increases, the flow penetration gradually comes to vertical and therefore, horizontal and vertical penetration of the flow almost the same. At the low Rayleigh number, the movement of the flow at the right corner of the enclosure is very high when compared to the movement of the flow at the left corner. As the Rayleigh number increases, movement of the flow at the left corner and at the right corner almost the same and therefore, flow penetration gradually increases with the Rayleigh number. Another observation is that the absolute values of vorticity increases as the Rayleigh number increases and hence the rotation of convective cell increases with the Rayleigh number. One vortex is observed for the Rayleigh number $10^{5}$ while the two counter rotating large vortices are predicted at the high Rayleigh number.

In the aperture plane, strength of circulation is 0.0098 $(x=0.58, y=0.4)$ at the Rayleigh number $10^{4}$. As the Rayleigh number increases from $10^{5}$ to $4.9 \times 10^{5}$, strength of circulation increases from $0.05925(x=0.58, y=0.4)$ to $0.271(x=0.5835, y=0.4)$ correspondingly.

For the aspect ratio 1.5 , at the Rayleigh number $10^{4}$, circulation strength is weak with $\left|\psi_{\text {ext }}\right|=0.00085$ at $x=$ $0.401, y=0.603$. As the Rayleigh number increases to $10^{5}$, correspondingly the location changes to $x=0.401, y=$ 0.604 with strength 0.0091 and penetration into the hump region increases. Further increase in the Rayleigh number results gradually full penetration into the hump with circulation strength $0.1068\left(\mathrm{Ra}=6.6 \times 10^{5}\right)$ at the location $x$ $=0.05$ and $y=0.603$. Also, it is observed that the temperature gradient is a decreasing function of the Rayleigh number along the symmetry plane. The reason being (1) formation of the vortex a little far away from the surface involved in resisting heat transfer performance (2) penetrations from both horizontal and vertical directions are almost the same. Therefore, length of the symmetry plane participating in heat transfer to the right cold cell decreases.
In the aperture plane, circulation strength $0.0097(x=$ $0.579, y=0.4$ ) at the Rayleigh number $10^{4}$. As the Rayleigh number increases to $10^{5}$, location is moving towards inside with strength 0.055 and level of penetration of the fluid increases. At the high Rayleigh number achieved, circulation with maximum strength 0.289 at the coordinate $x=0.58, y=0.4$. By comparison of isotherms at the high Rayleigh number in terms of aspect ratio indicates that the heat transfer rate is more at the high aspect ratio.

Figure 3(b) shows temperature contours at the high Rayleigh number achieved for aspect ratios 0.5, 1 and 2 . Temperature boundary layer on the circumference of outer layer surface is studied. The surface temperature moves along the boundary layer in clockwise direction and entrained into the main stream at the position where in it encounters the temperature moves along the boundary layer in an anti-clockwise direction. The mechanism of heat transfer is by a single cell rotating in clockwise direction in the left half driven by hot surface in between 0.36 and 0.43 . The heat is transferred to the right half along the symmetry line at $x=0.435$, as a result of which a counter-clockwise rotating cell is formed. Inner cell to the left of the symmetry plane receives heat from the outer convective cell and therefore records highest temperature among hot convective cells while innermost cell to the right of symmetry plane losses heat to outer convective cells and hence records lowest temperature among cold convective cells.

In case of the aspect ratio 1 , temperature gradient along the symmetry plane $x=0.435$ increases as the Rayleigh number increases.

Therefore, thermal penetration is more intensive with higher temperature gradient along the symmetry plane, (a) $\operatorname{ar}=1$

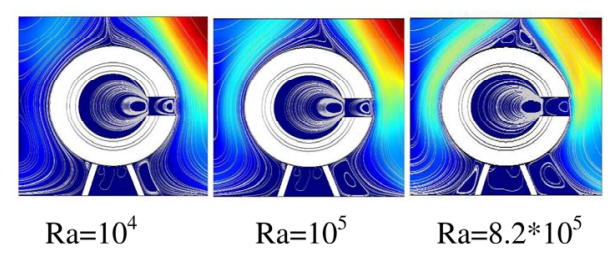

(b)

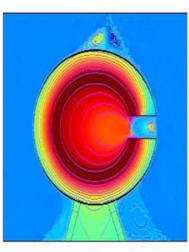

$\operatorname{ar}=1$

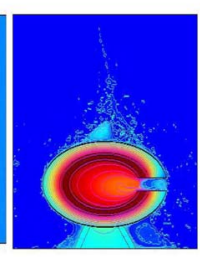

$\operatorname{ar}=0.5$

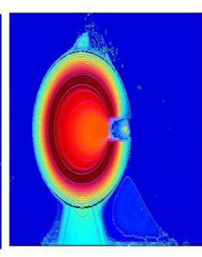

$\operatorname{ar}=1.5$
Figure 3. (a) Stream line plot for the aspect ratio 1; (b) Isothermal plots at high Rayleigh numbers. 
i.e., increasing heat transfer from the hot convective cell to the cold convective cell. From the plot, as the Rayleigh number increases, thermal penetration shift towards right while symmetry line shift towards left. Though weak cells are generated beyond penetration limit at low Rayleigh numbers, disappears as the Rayleigh number increases.

In the aperture zone, the fluid flow is stagnant in between $x=0.5$ and $x=0.51$ while the fluid flow penetrating through the aperture. Therefore, high temperature field is predicted in between $x=0.5$ and $x=0.51$. As the Rayleigh number increases, the size of stagnant flow is slightly reduced. From the temperature contours plot, isotherms bend like inverted parabolas leave the aperture toward inner surface of the cavity.

\subsection{Free Convection in the Hump Regime}

Local Nusselt number distribution along the horizontal layer of cross section near the proximity of surface top for various values of the aspect ratio $0.5,1$ and 1.5 and for various values of Rayleigh number studied. For the aspect ratio 1, maximum of local Nusselt number increases as one moves from surface top to the environment. It indicates that heat transfer from the surface to the ambient medium decreases. As the Rayleigh number increases to $8.2 \times 10^{5}$, maximum of local Nusselt number decreases as one move away from the surface.

Therefore, heat transfer from surface to ambient increases. Also, heat transfer behavior is same qualitatively for the aspect ratio 0.5 and 1 .

Figure 4 shows the local Nusselt number along the symmetry line and the line passes through hot convective cells at the high Rayleigh number $8.2 \times 10^{5}$ for the aspect ratio 1 . From the plot, it clears that Nusselt number close to zero at two instances along the symmetry plane while it is almost zero at five positions along the line passes through the hot cells. One important observation is that number of positions at which Nusselt number proximity

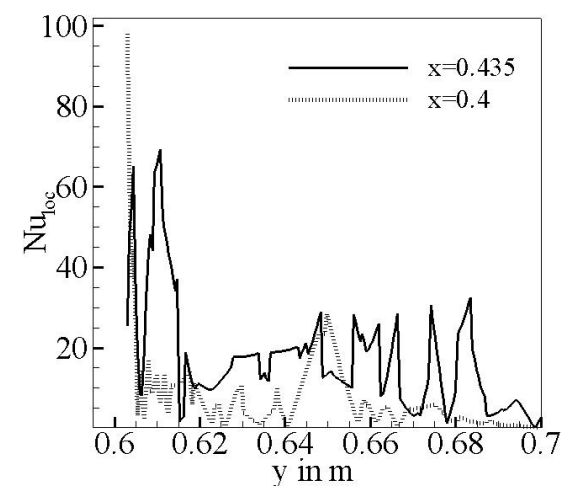

Figure 4. Local Nusselt number along the symmetry line and the line touches hot convective cell at $x=0.4$ and $x=$ 0.435 . to zero represent the number of cells the line touches.

\subsection{Free Convection in the Aperture Zone}

In the aperture zone, local Nusselt number distribution along the horizontal layer of cross section is studied for various values of the aspect ratio 0.5, 1 and 1.5. Figure 5 shows the graph of local Nusselt number against Rayleigh number in the aperture plane for the aspect ratio 1 . From the plot, it clears that convection is gradually dominated and therefore penetration of cold fluid into the aperture increases as the Rayleigh number increases to $8.2 \times 10^{5}$. Another important observation is that many weak convective cells are observed at Rayleigh number $10^{4}$. As the Rayleigh number increases, weak cells vanish and converted to very few strong convective cells inside the plane.

Figure 6 shows thermal penetration is $100 \%$ in the aperture plane at the high aspect ratio 1.5. Another observation is that number of strong convective cells increases as the aspect ratio increases from 0.5 to 1 .

\section{Conclusions}

In view of results and discussions presented, the following main conclusions are drawn.

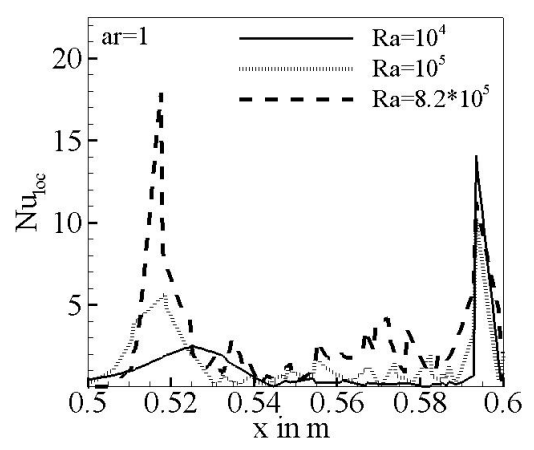

Figure 5. Local Nusselt number distribution along horizontal layer of cross section in the aperture zone for Rayleigh numbers $10^{4}, 10^{5}$ and $8.2 \times 10^{5}$.

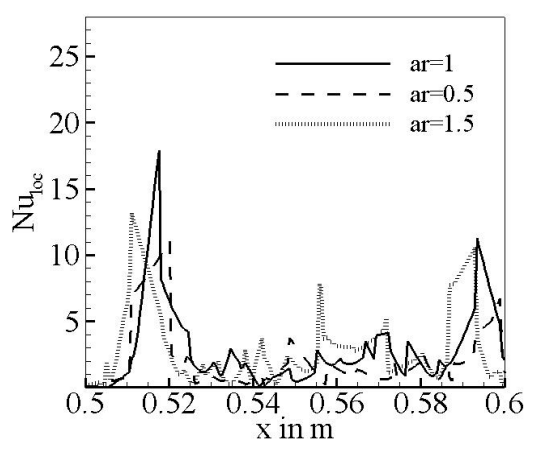

Figure 6. Local Nusselt number distribution along horizontal layer of cross section in the aperture for the aspect ratios $0.5,1$ and 1.5 . 
- Circulation strength has determined at the stagnant fluid and concluded that strength of the circulation increases with the Rayleigh number and the aspect ratio.

- Penetrative convection is more at the high aspect ratio.

- Enclosure of the high aspect ratio has been suggested for stable solution.

- Solution has been optimized with the constraint $\mathrm{T}=$ $409.8^{\circ} \mathrm{C}$.

\section{Acknowledgements}

The corresponding author is indebted to Ministry of University and Research, Cassino, Italy for their financial support and thankful to Prof. Marco dell'Isola for thorough discussions on the calibration furnace.

\section{REFERENCES}

[1] D. Nutter and D. P. Dewitt, "Theory and Practice of Radiation Thermometry," Wiley-Interscience, New York, 1989.

[2] S. Matle and S. Sundar, “Axi Symmetric 2D Simulation and Numerical Heat Transfer Characteristics for the Calibration Furnace in a Rectangular Enclosure,” Applied Mathematical Modeling, Vol. 36, No. 3, 2012, pp. 878893. doi:10.1016/j.apm.2011.07.047

[3] S. J. Cogan, K. Ryan and G. J. Sheard, "The Effects of Vortex Breakdown Bubbles in the Mixing Environment inside a Base Driven Bioreactor,” Applied Mathematical Modeling, Vol. 35, No. 4, 2011, pp. 1628-1637.

[4] A. Riahi and J. H. Curvan, "Full 3D Finite Element Cosserat Formulation with Application in Layered Structures," Applied Mathematical Modeling, Vol. 33, No. 8, 2009, pp. 3450-3464. doi:10.1016/j.apm.2008.11.022

[5] A. Prhashanna and R. P. Chhabra, "Free Convection in Power-Law Fluids from a Heated Sphere," Chemical Engineering Science, Vol. 65, No. 23, 2010, pp. 6190-6205. doi:10.1016/j.ces.2010.09.003

[6] M. Z. Salleh, R. Nazar and I. Pop, "Modelling of Free Convection Boundary Layer Flow on a Solid Sphere with Newtonian Heating," Acta Applicandae Mathematicae, Vol. 112, No. 3, 2010, pp. 263-274. doi:10.1007/s10440-010-9567-5

[7] O. O. Oluwole, P. O. Atanda and B. I. Imasogie, "Finite Element Modeling of Heat Transfer in Salt Bath Furnaces," Journal of Minerals and Materials Characterization and Engineering, Vol. 8, No. 3, 2009, pp. 209-236.

[8] A. R. Khoei, I. Masters and D. T. Gethin, "Numerical Modeling of the Rotary Furnace in Aluminium Recycling
Processes," Journal of Materials Processing and Technology, Vol. 139, No. 1-3, 2003, pp. 567-572. doi:10.1016/S0924-0136(03)00538-7

[9] P. A. B. de Sampaio, P. R. M. Lyra, K. Morgan and N. P. Weatherill, "Petrov-Galerkin Solutions of the Incompressible Navier-Stokes Equations in Primitive Variables with Adaptive Remeshing," Computer Methods in Applied Mechanics and Engineering, Vol. 106, No. 1-2, 1993, pp. 143-178. doi:10.1016/0045-7825(93)90189-5

[10] W. N. R. Stevens, "Finite Element Stream Function-Vorticity Solution of Steady Laminar Natural Convection," International Journal for Numerical Methods in Fluids, Vol. 2, No. 4, 1982, pp. 349-366. doi:10.1002/fld.1650020404

[11] I. Goldhirsch, R. B. Pelz and S. A. Orszang, "Numerical Simulation of Thermal Convection in a Two Dimensional Finite Box,” Journal of Fluid Mechanics, Vol. 199, 1989, pp. 1-28. doi:10.1017/S0022112089000273

[12] D. E. Fitzjarrald, “An Experiment Study of Turbulent Convection in Air,” Journal of Fluid Mechanics, Vol. 73, No. 4, 1976, pp. 337-353.

[13] E. Bilgen and R. B. Yedder, "Natual Convection in Enclosure with Heating and Cooling by Sinusoidal Temperature Profiles on One Side," International Journal of Heat and Mass Transfer, Vol. 50, No. 1-2, 2007, pp. 139150. doi:10.1016/j.ijheatmasstransfer.2006.06.027

[14] I. E. Sarris, I. Lekakis and N. S. Vlachos, "Natural Convection in a 2D Enclosure with Sinusoidal Wall Temperature,” Numerical Heat Transfer, Part A: Applications: An International Journal of Computation and Methodology, Vol. 42, No. 5, 2002, pp. 513-530. doi:10.1080/10407780290059675

[15] T. Hartlep, A. Tilgner and F. H. Busse, "Large Scale Structures in Rayleigh-Benaurd Convection at High Rayleigh Numbers,” Physical Review Letters, Vol. 91, No. 6, 2000. doi:10.1103/physRevLett.91.064501

[16] J. R. Lee, M. Y. Ha and S. Balachander, "Natural Convection in a Horizontal Fluid Layer with a Periodic Array of Internal Square Cylinders-Need for Very Large Aspect Ratio Domains," International Journal of Heat and Fluid Flow, Vol. 28, No. 5, 2007, pp. 978-987. doi:10.1016/j.ijheatfluidflow.2007.01.005

[17] R. Becker and B. Vexler, “A Posteriori Error Estimation for Finite Element Discretizations of Parameter Identification Problems,” Numerische Mathamatics, Vol. 96, No. 3, 2004, pp. 1-102.

[18] R. Becker and B. Vexler, "Mesh Refinement and Numerical Sensitivity Analysis for Parameter Calibration of Partial Differential Equations,” Journal of Computational Physics, Vol. 206, No. 1, 2005, pp. 95-110. doi:10.1016/j.jcp.2004.12.018 


\section{Nomenclature}

$x, y$ Cartesian coordinates

$u, v$ Velocity vectors

$p$ Pressure

$T$ Solution temperature, $\left[{ }^{\circ} \mathrm{C}\right]$

$R a$ Rayleigh number

$g$ Acceleration due to gravity, $\left[\mathrm{m}^{2} / \mathrm{s}\right]$

$\kappa$ Thermal conductivity $[\mathrm{W} / \mathrm{mK}]$

$P r \quad$ Prandtl number $=0.71$

$Q$ Heat source $\left[\mathrm{W} / \mathrm{m}^{3}\right]$

\section{Greeks}

$\alpha$ Thermal diffusivity, $\left[\mathrm{m}^{2} / \mathrm{s}\right]$

$\beta$ Coefficient of thermal expansion, $[1 / K]$

$C_{p}$ Specific heat capacity at constant pressure, $[\mathrm{J} / \mathrm{KgK}]$

$\rho$ Density of air, $\left[\mathrm{kg} / \mathrm{m}^{3}\right]$

$\varepsilon \quad$ Emissivity of the cavity $=0.98$

$\theta \quad$ Dimensionless temperature $=\left(T-T_{\text {ref }}\right) / \Delta T$ 